\title{
Phylogeny of Theileria buffeli genotypes identified in the South African buffalo \\ (Syncerus caffer) population
}

\author{
Mamohale E. Chaisi ${ }^{\text {a,b, }}$, Nicola E. Collins ${ }^{\mathrm{a}}$, Marinda C. Oosthuizen ${ }^{\mathrm{a}}$ \\ ${ }^{a}$ Department of Veterinary Tropical Diseases, Faculty of Veterinary Science, University of Pretoria, Private Bag X04, \\ Onderstepoort, 0110, South Africa \\ ${ }^{\mathrm{b}}$ Department of Biology, National University of Lesotho, Faculty of Science and Technology, Roma 180, Lesotho; \\ * Corresponding author. Tel. +27 735715876; fax: +27 125298312. \\ E-mail address: mechaisi@yahoo.co.uk (M.E. Chaisi)
}

\begin{abstract}
Theileria buffeli/T. sergenti/T. orientalis is a group of benign and mildly pathogenic species of cattle and buffalo. In a previous study, we identified $T$.buffeli in blood samples originating from the African buffalo (Syncerus caffer) in the Hluhluwe-iMfolozi Game Park (HIP) and the Addo Elephant Game Park (AEGP) in South Africa. The aim of this study was to characterize the $18 \mathrm{~S}$ rRNA gene and complete internal transcribed spacer (ITS1-5.8S-ITS2) region of T. buffeli samples, and to establish the phylogenetic position of this species based on these loci. The 18S rRNA gene and the complete ITS region were amplified from DNA extracted from blood samples originating from buffalo in these localities. The PCR products were cloned and the resulting recombinants sequenced. We identified novel T. buffeli $18 \mathrm{~S}$ rRNA gene and ITS genotypes from buffalo in the AEGP, and novel T. sinensis 18S rRNA genotypes from buffalo in the HIP. Phylogenetic analyses indicated that the T. buffeli-like sequences were similar to T. buffeli sequences from cattle and buffalo in China and India, and the $T$. sinensis-like sequences were similar to $T$. sinensis $18 \mathrm{~S}$ rRNA sequences of cattle and yak in China. There was extensive sequence variation between the novel $T$.
\end{abstract}


buffeli genotypes of the African buffalo and previously described $T$. buffeli and $T$. sinensis genotypes. The presence of organisms with $T$. buffeli-like and $T$. sinensis-like genotypes in the African buffalo could be of significant importance, particularly to the cattle industry in South Africa as these animals might act as sources of infections to naïve cattle. This is the first report on the characterization of the full-length $18 \mathrm{~S}$ rRNA gene and ITS region of T. buffeli and T. sinensis genotypes in South Africa. Our study provides invaluable information towards the classification of this complex group of benign and mildly pathogenic species.

Keywords: T. buffeli/T. sergenti/T. orientalis; African buffalo; 18S rRNA; ITS

\section{Introduction}

Theileria buffeli/Theileria sergenti/Theileria orientalis is a group of closely related parasites of cattle and buffalo. They have a cosmopolitan distribution and infect cattle and buffalo in Africa, Australia, Asia, Europe and the United States of America (USA) (Chae et al., 1998; Chansiri et al., 1999; Cossio-Bayugar et al., 2002; Sarataphan et al., 2003; Aktas et al., 2007; Altay et al., 2008; M'ghirbi et al., 2008; Gimenez et al., 2009, Liu et al., 2010a, Wang et al., 2010). The cosmopolitan distribution of these species has been attributed to the global movement of cattle (and buffalo) without any regard to infection, and therefore their distribution mainly depends on the availability of a suitable tick vector (Chae et al., 1999c; Cossio-Bayugar et al., 2002). Haemaphysalis ticks act as vectors in Australia, Asia and Europe, but the vectors in Africa and the USA are still unknown (Yin et al., 2004; Bendele, 2005; M'ghirbi et al., 2008). Benign isolates from Britain, Australia and the USA were initially designated as T. mutans as their pathology was similar to that of $T$. mutans (Chae et al., 1999c). However, further studies indicated that T. mutans is an African species and is serologically and genetically distinct from other benign Theileria spp. (Morzaria et al., 1977, Chae et al., 1999c). 
Theileria sergenti and T. orientalis were first described from eastern Siberia in the early 1930s by, respectively, Yakimoff and Dekhtereff, and Yakimoff and Soudatschenkoff, while T. buffeli was first described from the Asian water buffalo (Bubalus bubalis) in 1908 by Schein (reviewed by Fujisaki et al., 1994). The classification of these benign parasites is still confusing and is complicated by their similar morphology, serology, vector transmission, geographical distribution, difficulties in obtaining pure isolates and incompletely understood life-cycles (Uilenberg et al., 1985; Chae et al., 1999c; Chansiri et al., 1999; Yin et al., 2004; M'ghirbi et al., 2008, Uilenberg, 2011). It is still unclear if these organisms represent the same species or different species. However, T. sergenti is pathogenic to cattle and yak, and is regarded as a separate species from the benign $T$. buffeli/T. orientalis (Kawazu et al., 1999). Other authors (Fuujisaki, 1992; Chae et al., 1999a; Uilenberg, 2011) indicated that although the term "T. sergenti" has traditionally been used for this species, $T$. sergenti actually refers to a sheep parasite and was incorrectly used to name a parasite of cattle and buffalo. Due to all this confusion, Uilenberg et al. (1985) suggested that the benign species (T. buffeli/T. orientalis) should be classified as T. orientalis. However, the term T. buffeli is preferred over $T$. orientalis on the basis of molecular data, as well as the fact that all characterized isolates are infective for buffalo (Steward et al., 1996). Gubbels et al. (2000) therefore proposed that these organisms should be referred to as T. buffeli until more biological data becomes available for further classification, and the names $T$. orientalis and $T$. sergenti should only refer to isolates that have been previously described under these names.

Another closely related species, Theileria sinensis, was recently described in China and is also regarded as a cause of bovine theileriosis in that country (Bai et al., 2002a; b, cited by Yin et al., 2004). T. sinensis is transmitted by Haemaphysalis qinghaiensis ticks and infects cattle, yak (Yin et al., 2002; 2004; Sun et al., 2008) and water buffalo (He et al., 2012) in China.

Molecular biology studies based on the $18 \mathrm{~S}$ ribosomal RNA (rRNA) gene, internal transcribed spacers (ITS), major piroplasm surface protein (MPSP) gene and other genetic markers have 
provided useful information on the epidemiology, diagnosis, taxonomy and phylogeny of these benign Theileria spp. (Allsopp et al., 1994; Chae et al., 1998, Chansiri et al., 1999; Gubbels et al., 2000, 2002; Sarataphan et al., 2003; M'ghirbi et al., 2008; Liu et al., 2010a; b; Wang et al., 2010; Kamau et al., 2011). We recently identified T .buffeli in some buffalo populations in South Africa (Chaisi et al., 2011). Although Mans et al. (2011) previously characterized the V4 hypervariable region of the $18 \mathrm{~S}$ rRNA gene of $T$. buffeli of cattle and buffalo originating from different geographical regions of South Africa, there is still a need to determine their phylogenetic positions based on other genetic markers.

The ribosomal ITS region in eukaryotes is located between the small (18S) and large (28S) subunits of the ribosomal RNA gene, and spans the two ribosomal RNA transcribed spacers and the 5.8S gene (ITS1-5.8S-ITS2) (Aktas et al., 2007). Unlike the rRNA genes which are highly conserved between closely related species, the spacer regions (ITS1 and ITS2) are subject to higher evolutionary rates and are therefore more variable in their lengths and nucleotide composition (Hillis and Dixon, 1991). These regions have therefore been used for the discrimination of closely related species and subspecies, and in the description of new species (Zahler et al., 1998; Holman et al., 2003; Lew et al., 2003; Aktas et al., 2007; de Rojas et al., 2007; Hilpertshauser et al., 2007; Saito-Ito et al., 2008; Niu et al., 2009; Bosman et al., 2010).

The aims of this study were to: (1) sequence the full-length 18S rRNA gene and complete ITS (ITS1-5.8S-ITS2) region of T. buffeli of the South African buffalo; (2) determine the level of genetic variation between novel T. buffeli-like and T. sinensis-like genotypes of the African buffalo with known T. buffeli and T. sinensis genotypes; and (3) to establish their phylogenetic positions based on their full-length 18S rRNA gene and complete ITS sequences. 


\section{Materials and Methods}

\subsection{DNA samples}

A molecular epidemiological survey based on the 18S rRNA gene was previously carried out to determine the occurrence of Theileria spp. from the African buffalo in different geographic areas in South Africa and Mozambique using the reverse line blot (RLB) hybridization assay (Chaisi et al., 2011). In that study, Theileria buffeli was identified from buffalo blood samples originating from the Hluhluwe-iMfolozi Game Park (HIP). Based on the RLB results, four samples (HIP/A2, HIP/A4, HIP/C5, HIP/C23) were selected for characterization of their full-length 18S rRNA genes, while the complete ITS (ITS1-5.8S-ITS2) region was characterized from ten samples (HIP/A36, HIP/B62, HIP/C11, HIP/C13, HIP/C15, HIP/C18, HIP/C19, HIP/C23, HIP/C25, HIP/C27). Theileria buffeli is also known to occur in buffalo in the Addo Elephant Game Park (AEGP), Eastern Cape Province, South Africa. The parasite 18S rRNA gene and ITS region were also characterized from seven samples originating from buffalo in the AEGP (AEGP/65, AEGP/66, AEGP/69, AEGP/70, AEGP/73, AEGP/74, AEGP/76). T. buffeli is the only Theileria spp. known to infect buffalo in the AEGP, and only T. buffeli was identified in the AEGP samples using the RLB (Milana Troskie, personal communication). The samples from the HIP contained mixed Theileria spp. infections (Chaisi et al. (2011).

2.2 Amplification, cloning and sequencing of the full-length $18 S$ rRNA gene and complete ITS (ITS1-5.8S-ITS2) region

The full length 18S rRNA genes of 11 samples ( 4 from HIP and 7 from AEGP) were amplified by conventional PCR using forward primer Nbab-1F and reverse primer 18SRev-TB (Oosthuizen et al., 2008). The reaction mixture and cycling conditions were as described by Chaisi et al. (2011). The resulting amplicons were purified using the QIAquick PCR Purification Kit (Qiagen, Southern Cross Biotechnologies).

A nested PCR protocol was used to amplify the complete parasite ITS region of 17 samples (10 from HIP and 7 from AEGP). The primary reaction contained $2.5 \mu \mathrm{l}(\sim 75 \mathrm{ng})$ genomic DNA, 0.1 $\mu \mathrm{M}$ each of primer 1055F (5'- GGT GGT GCA TGG CCG-3') and LSUR300 (5' $-\mathrm{T}(\mathrm{A} / \mathrm{T}) \mathrm{G}$ CGC 
TTC AAT CCC-3') (Holman et al., 2003; Aktas et al., 2007), 1.5 mM MgCl $2,200 \mu \mathrm{M}$ dNTPs, High Fidelity Enzyme blend (Roche Diagnostics, Mannheim, Germany) and nuclease-free water to a total volume of $25 \mu \mathrm{l}$. The thermal cycling programme was done at an initial denaturation at $96^{\circ} \mathrm{C}$ for 3 min, followed by 30 cycles of denaturation at $94^{\circ} \mathrm{C}$ for $30 \mathrm{~s}$; annealing at $50^{\circ} \mathrm{C}$ for $30 \mathrm{~s}$; extension at $72^{\circ} \mathrm{C}$ for $3 \mathrm{~min}$; a final extension at $72^{\circ} \mathrm{C}$ for $7 \mathrm{~min}$ and then hold at $4^{\circ} \mathrm{C}$. Primers ITSF $\left(5^{\prime}-\mathrm{GAG}\right.$ AAG TCG TAA CAA GGT TTC CG-3') and LSUR50 (5'-GCT TCA CTC GCC GTT ACT AGG3') (Holman et al., 2003) were used for the nested PCR. The reaction mixture was as above, except that $1 \mu \mathrm{l}(\sim 30 \mathrm{ng})$ of the primary PCR product was used as template. The cycling conditions were also as above, except that annealing was done at $60^{\circ} \mathrm{C}$ for $30 \mathrm{~s}$ and extension was done at $72^{\circ} \mathrm{C}$ for 2 $\min$.

Amplicons of four reactions per sample were pooled to avoid Taq polymerase induced errors. For the HIP samples, which all contained mixed Theileria spp. infections and therefore could not be directly sequenced, purified 18S rRNA and ITS amplicons were ligated into the pGEM-T Easy Vector and transformed into E. coli JM109 High Efficiency Competent cells (Promega, Madison, WI). At least 5 positive white colonies were selected per sample. Recombinant plasmid DNA was extracted from overnight bacterial cultures using the High Pure Plasmid Isolation kit (Roche Diagnostics, Mannheim, Germany).

Sequencing of the $18 \mathrm{~S}$ rRNA genes and complete ITS region were done at the OVI and Inqaba Biotech respectively. Since $T$. buffeli is the only Theileria spp. known to infect buffalo in the AEGP, the amplicons obtained from samples from this game park were directly sequenced. However, the samples from the HIP were cloned prior to sequencing as mixed Theileria spp. infections are common in buffalo from this game park. The plasmids were initially screened by sequencing using the ABI BigDye ${ }^{\mathrm{TM}}$ Terminator Cycle Sequencing Ready Reaction kit (PE Applied Biosystems), 350 ng plasmid DNA and 3.2 pmol of primer RLB-F2 (Nijhof et al., 2003) for the 18S rRNA. The obtained sequences were subjected to a BLASTn (Altschul et al., 1990) similarity search. The full-length $18 \mathrm{~S}$ rRNA genes of recombinants with sequences that were closely similar 
to the published 18S rRNA gene sequences of $T$. buffeli or $T$. sinensis were subsequently sequenced using primers Nbab-1F, 18SRev-TB, RLB-R2, BT18S-2F, BT18S-3F, BT18S-4F, BT18S-4R, SP6, T7 (Osthuizen et al, 2008; Chaisi et al., 2011). For samples from the AEGP, which had single Theileria species infections, full-length $18 \mathrm{~S}$ rRNA genes were directly sequenced using ABI BigDye $^{\mathrm{TM}}$ Terminator Cycle Sequencing Ready Reaction kit (PE Applied Biosystems), 40 ng of PCR product and 3.2 pmol of each primer. Sequencing was done on an ABI3100 genetic analyzer at the sequencing facility of the Agricultural Research Council-Onderstepoort Veterinary Institute (ARC-OVI), South Africa.

For the complete ITS region, amplicons of approximately 1200 bp were excised from ethidiumbromide stained gels and purified using the Qiaquick Gel Extraction Kit (Qiagen, Southern Cross Biotechnologies), after which they were directly ligated into the pGEM-T Easy Vector and transformed into E. coli JM109 High Efficiency Competent cells (Promega, Madison, WI). Sequencing reactions were done using the ABI BigDye ${ }^{\mathrm{TM}}$ Terminator Cycle Sequencing Ready Reaction kit (PE Applied Biosystems), 300 ng of plasmid DNA and 2 pmol each of primers ITSF, SP6 and T7. Sequencing was done at Inqaba Biotechnologies, South Africa. The reactions were purified by the Zymo research sequencing clean-up kit (Inqaba Biotechnogies, South Africa) according to the manufacturer's protocol, and analysed with an ABI 3500XL genetic analyzer.

\subsection{Sequence and phylogenetic analyses}

The sequences were assembled and edited using the GAP4 program of the Staden package (version 1.6.0 for Windows) (Bonfield et al., 1995; Staden et al., 2000). A BLASTn homology search of GenBank was done using the full length consensus sequences. These were then aligned with $18 \mathrm{~S}$ rRNA gene sequences (Table 1) or ITS sequences of related genera from GenBank using the MAFFT (multiple sequence alignment) v6 programme employing the FFT-NS-1 algorithm (Katoh et al., 2005). The alignments were manually examined and edited across their full-lengths, and then truncated to the size of the smallest sequence using BioEdit v7 (Hall, 1999). Sequences with crossover sequences PCR or sequencing-induced artifacts (Thompson et al., 2002) were eliminated from 
the alignments. A total of 58 (new and known) 18S rRNA gene sequences (1514 bp), and 30 ITS sequences (1510 bp) were analysed. Estimated evolutionary divergence was calculated by determining the number of nucleotide differences between similar sequences over a region of 1499 and 1215 nucleotides for the 18S rRNA gene and ITS sequences, respectively. Nucleotide differences were also assessed in the V4 hypervariable region of the $18 \mathrm{~S}$ rRNA sequences, and in the ITS1, 5.8S gene and ITS2 regions of the ITS sequences.

Phylogenetic trees were inferred from the alignments by the neighbor-joining method (Saitou and Nei, 1987), maximum parsimony and maximum likelihood methods using PAUP* v4b10 (Swofford, 2003). These were done in combination with bootstrapping (1000 replicates). Bayesian inference was done using MrBayes v3.1.2 (Ronquist and Huelsenbeck, 2003), accessed via the Computational Biology Service Unit, Cornell University. For comparison of the phylogenetic trees, 18S rRNA gene and ITS sequences of the same species or isolate were included, where possible. The 18S rRNA gene and ITS sequences of Babesia canis, B. caballi and B. orientalis were included as outgroups to root the phylogenetic trees. All consensus trees were edited using MEGA4 (Tamura et al., 2007).

The near full-length 18S rRNA gene sequences have been deposited in GenBank under accession numbers JQ037779 - JQ037790. The complete ITS sequences have been deposited in GenBank under accession numbers JQ0377791 - JQ037795.

\section{Results}

\subsection{Identification of T. buffeli-like and T. sinensis-like $18 S$ rRNA gene sequences}

Single bands of approximately $1700 \mathrm{bp}$, as viewed on a $2 \%$ ethidium bromide stained agarose gel, were obtained. These were cloned and sequenced. A total of 12 (5 from HIP and 7 from AEGP) near full-length 18S rRNA sequences were obtained (Table 1). The lengths of the HIP sequences 
(GenBank accession nos. $\underline{\mathbf{J Q 0 3 7 7 8 6}}, \underline{\mathbf{J Q 0 3 7 7 8 7}}, \underline{\mathbf{J Q 0 3 7 7 8 8}}, \underline{\mathbf{J Q 0 3 7 7 8 9}}, \underline{\mathbf{J Q 0 3 7 7 9 0}}$ ) ranged from 1582 - 1592 bp. (Table 2). A BLASTn homology search did not reveal any identical sequences in GenBank, the closest homology (98\% and 99\%) was found with 18S rRNA gene sequences of

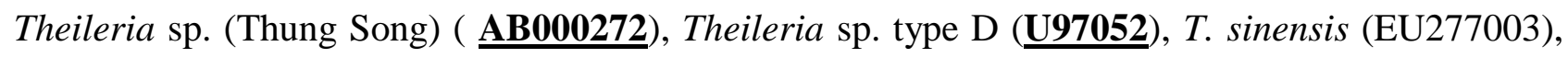

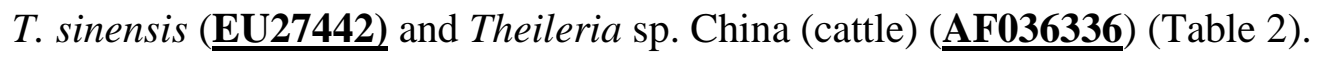

The lengths of the seven 18S rRNA sequences from AEGP (GenBank accession nos $\underline{\mathbf{J Q 0 3 7 7 7 9}}$, $\underline{\mathbf{J Q 0 3 7 7 8 0}}, \underline{\mathbf{J Q 0 3 7 7 8 1}}, \underline{\mathbf{J Q 0 3 7 7 8 2}}, \underline{\mathbf{J Q 0 3 7 7 8 5}}, \underline{\mathbf{J Q 0 3 7 7 8 3}}, \underline{\mathbf{J Q 0 3 7 7 8 4}})$ were 1587 - 1588 bp. BLASTn similarity searches of these sequences did not reveal any identical sequences, but they were most similar (99\%) to T. buffeli 18S rRNA gene sequences from China (DQ104611 and $\underline{\text { HM538212 }})$ and India (ㅌ126184).

\subsection{Sequence and phylogenetic analyses of the 18S rRNA genes}

The T. buffeli sequences formed 9 distinct clusters, and were clearly separated from other Theileria spp. (Figure 1). The clustering was similar in all trees, but there were differences in the branching of the clusters in some trees. Six of the nine T. buffeli genotypes (designated Types A, B/Ikeda, C/Medan, D, E/H/Ipoh, Warwick) (Figure 2) are known T. buffeli 18S rRNA genotypes (Chae et al., 1998; 1999a; Chansiri et al., 1999; Gubbels et al., 2000; Yin et al., 2004). Types F and G are T. cervi $18 \mathrm{~S}$ rRNA sequences from the elk and white-tailed deer in the USA and Canada (Chae et al., 1999c) and are distantly related to the T. buffeli genotypes (Figure 1).

Sequence and phylogenetic analyses indicated the presence of one more known, but unclassified $T$. buffeli genotype from China (Liu et al., 2010a) and India (unpublished), and two novel T. buffelilike genotypes from South Africa which we designated as types SA1 and SA2 (Figure 1). Genotype SA1 is composed of $18 \mathrm{~S}$ rRNA gene sequences originating from the AEGP, and the sequences of this group are closely related to those of the unclassified group (DQ104611, $\underline{\text { HM538212}}$, EF126184). Genotype SA2 is composed of $18 \mathrm{~S}$ rRNA gene sequences from the HIP, and is closely related to the $T$. buffeli type $\mathrm{D} / T$. sinensis group (Figure 1). 


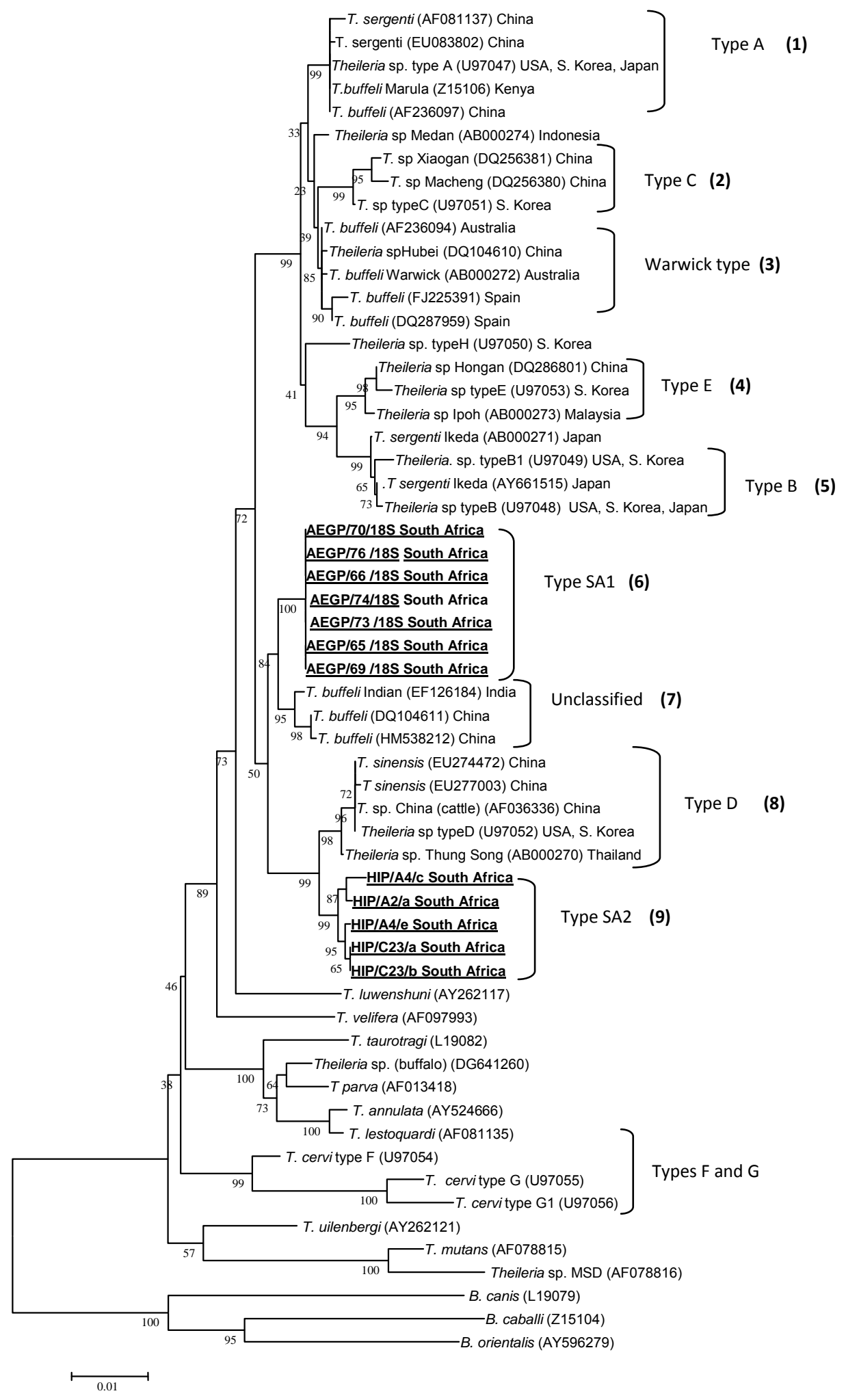

Figure 1: Phylogenetic relationships of known T. buffeli sequences with novel T. buffeli-like and T.sinensis-like 18S rRNA gene sequences from South Africa (underlined), as inferred by the Neighbor-joining method. GenBank accession numbers are indicated in parenthesis. Numbers In brackets are designated cluster numbers. Bootstrap values are indicated at the nodes. 
In order to estimate the genetic distance between the $T$. buffeli-like sequences, the novel genotypes were aligned with 17 known $T$. buffeli $18 \mathrm{~S}$ rRNA gene sequences (representing the different genotypes), and compared along a region of $1499 \mathrm{bp}$. Sequence variation was observed both within and between the different $T$. buffeli genotypes. All seven novel sequences from AEGP were identical within this region and along their full lengths (results not shown). These sequences differed from those of the closely related genotype (unclassified) by $9-12 \mathrm{bp}$, and from the novel sequences from HIP by $21-23$ bp (Table 3). The HIP sequences differed from the T. buffeli type D/T. sinensis sequences by 11 - 16 bp. Sequences HIP/C23/a and HIP/C23/b were identical, while there was a 7 bp difference between sequences HIP/A4/c and HIP/A4/e. The other novel sequences from HIP differed from each other by $2-7 \mathrm{bp}$. The greatest variation ( $45 \mathrm{bp})$ was observed between sequence HIP/A4/c and Theileria sp. type E, which is from cattle isolates in the USA and South Korea (Chae et al., 1998).

Most of the variation between $T$. buffeli-like genotypes occurred in the V1 variable region (positions 70 - 140) and V4 hypervariable region (positions 490 - 560) of the gene (Figures 2A and B). Figure 2B shows the positions of the RLB probes that were designed for the detection of all $T$. buffeli 18S rRNA gene sequences (Gubbels et al., 1999) and the specific T. buffeli probe (Gubbels et al., 2000).

\subsection{Sequence and phylogenetic analyses of the novel ITS sequences}

Amplicons of approximately $1200 \mathrm{bp}$, as viewed on a 2\% agarose gel, were observed from the nested PCR products. Eleven ITS sequences were obtained from the 10 samples from HIP. However, examination of the alignment of these sequences with published ITS sequences suggested that the new sequences were cross-over sequences (Thompson et al., 2002). They were therefore excluded from further analyses. 
Theileria sp. type A (U9704)*

T. buffeli Warwick (AB000272)

Theileria sp. Medan (AB000274)

T. sergenti (AB000271)

Theileria sp. typeB (U97048)

Theileria sp. typeB1 (U97049)

Theileria sp. typeE (U97053)

Theileria sp. typeC (U97051)

Theileria sp. typeH (U97050)

T. buffeli Indian (EF126184)

T. buffeli (DQ104611)

T. buffeli (HM538212)

AEGP / 65/18S

AEGP / 6 6/18S

AEGP/69/18S

$\mathrm{AEGP} / 70 / 18 \mathrm{~S}$

AEGP / 74/18S

AEGP / 76/18S

AEGP/73/18S

T sp. Thung Song (AB000270)

T. sp. China (cattle) (AF036336)

T. sinensis (EU274472)

Theileria sp. typed (U97052)

T. sinensis (EU277003)

HIP/A4/C

$\mathrm{HIP} / \mathrm{A} 4 / \mathrm{e}$

$\mathrm{HIP} / \mathrm{C} 23 / \mathrm{a}$

i $\mathrm{HIP} / \mathrm{C} 23 / \mathrm{b}$

$\mathrm{HIP} / \mathrm{A} 2 / \mathrm{a}$
ССTAAAACCAAACСTTTT-- $\ldots|\ldots| \ldots|\ldots| \ldots|\ldots| \ldots|\ldots| \ldots|\ldots| \ldots|\ldots| \ldots|\ldots|$

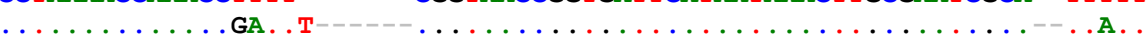

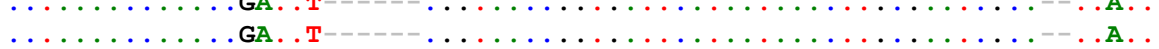

$\ldots \ldots \ldots \ldots$ T. G.GCCAAACA $\ldots$ TC . . . . . . . . . . . .

$\ldots \ldots \ldots$ T. . GCCAAACA $-\ldots$ TC. . . . . . . . . . . . . .

$\ldots \ldots \ldots$ T. . GCCAAACA $\ldots$ TC . . . . . . . . . . . . . .

$\ldots \ldots \ldots$. . . . . GCTTCTGCG. . TC . . . . . . . . . . . . . . . А

$\ldots \ldots$ G. . . . . GGG. TCTTCC $\ldots$ СTT. . . . . . . . . . . . . . . . . .

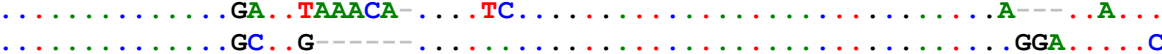

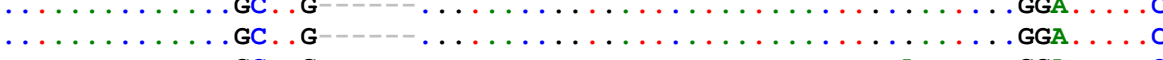

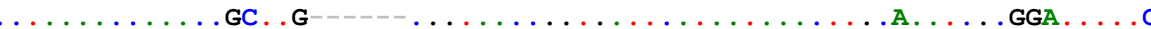

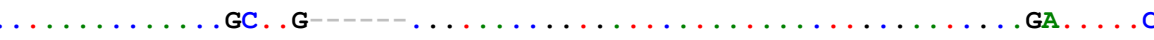

$\ldots \ldots \ldots \ldots \ldots \ldots \ldots \ldots \ldots \ldots \ldots \ldots \ldots \ldots \ldots \ldots \ldots \ldots \ldots \ldots \ldots$

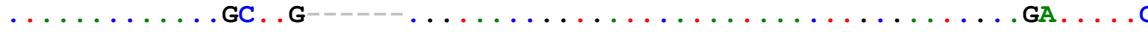

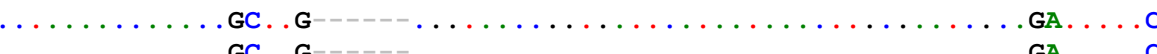

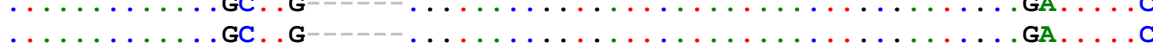

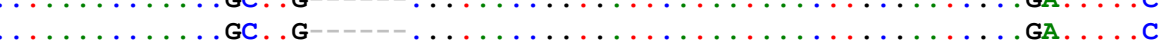

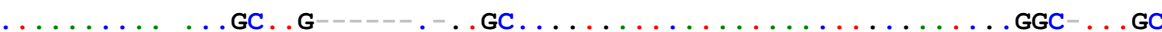

$\ldots \ldots \ldots \ldots \ldots$ GC G $_{-1} \ldots \ldots \ldots \ldots \ldots \ldots \ldots \ldots$ GGC $\ldots$ GC

$\ldots \ldots \ldots \ldots \ldots$ GC.$G^{---} \ldots \ldots$ GC $\ldots \ldots \ldots \ldots \ldots$ GGC $\ldots \ldots$ GC

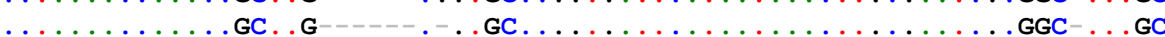

$\ldots \ldots \ldots \ldots \ldots$ GC. G $-\ldots \ldots \ldots \ldots \ldots \ldots \ldots \ldots \ldots \ldots$ GGC $\ldots \ldots$ GC

$\ldots \ldots \ldots \ldots \ldots$ GC. G

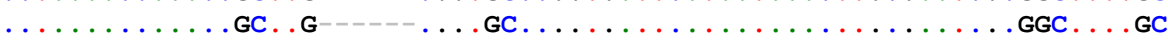

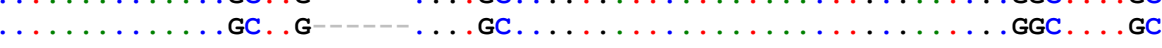

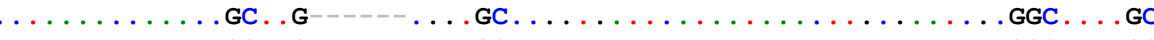

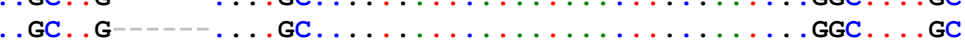

\begin{tabular}{llllll}
\hline B & 500 & 510 & 520 & 530 & 540
\end{tabular}

Theileria sp. type A (U9704)*

T. buffeli Warwick (AB000272)

Theileria sp. Medan (AB000274)

T. sergenti (AB000271)

Theileria sp. typeB (U97048)

Theileria sp. typeB1 (U97049)

Theileria sp. typeE (U97053)

Theileria sp. typec (U97051)

Theileria sp. typeH (U97050)

T. buffeli Indian (EF126184)

T. buffeli (DQ104611)

T. buffeli (HM538212)

AEGP/65/18S

AEGP/66/18S

AEGP $/ 69 / 18 \mathrm{~S}$

AEGP / 70/18S

AEGP/74/18S

AEGP $/ 76 / 18 \mathrm{~S}$

AEGP / 73/18S

T. sp Thung Song ( $\mathrm{AB} 000270$ )

T. sp. China (cattle) (AF036336)

T. sinensis (EU274472)

Theileria sp. typeD (U97052)

T. sinensis (EU277003)

$\mathrm{HIP} / \mathrm{A} 4 / \mathrm{C}$

$\mathrm{HIP} / \mathrm{A} 4 / \mathrm{e}$

$\mathrm{HIP} / \mathrm{C} 23 / \mathrm{a}$

HIP/C23/b

HIP/A2 /

$\ldots|\ldots| \ldots|\ldots| \ldots|\ldots| \ldots|\ldots| \ldots|\ldots| \ldots|\ldots| \ldots|\ldots| \ldots|\ldots|$ |

Ali T. buffeli (Gubbels et al., ig99)

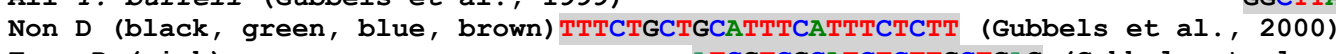

TTTCTGCTGCATTTCATTTCTCTT-TCTGAGTTGTTTTTGC\&GCTTATTTCGGTTTGA--TTTT中-TCT

\begin{tabular}{|c|c|c|}
\hline ЭСТGСАTTTCATTTCTCTT|TCTGAGTTTGTT & & \\
\hline$\ldots \ldots \ldots$. $\ldots$. & $\ldots \ldots \ldots \ldots$ & \\
\hline$\ldots \ldots \ldots$. $\ldots \ldots \ldots$. $\ldots \ldots \ldots$ А. & $\ldots \ldots \ldots$..... & A. \\
\hline$\ldots$ G........... & $\ldots \ldots \ldots$.... & A. \\
\hline G.T......... T & $\ldots \ldots \ldots$... & A. \\
\hline$\ldots \ldots \ldots$. $\ldots$. . . . . . G. TC . . . . A . . T & $\ldots \ldots \ldots$... & A. . A \\
\hline$\ldots \ldots \ldots$. & $\ldots \ldots \ldots$... & A... \\
\hline . G. . . . . . . . T & $\ldots \ldots \ldots \ldots$ & A. \\
\hline$\ldots \ldots \ldots \ldots \overline{\bar{A}} \ldots \ldots . \overline{\mathbf{A}} \ldots \ldots$ тстс . . . . & $\ldots \ldots \ldots \ldots \ldots$ & \\
\hline 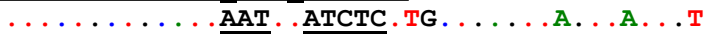 & $\ldots \ldots \ldots$.... & \\
\hline$\ldots \ldots \ldots \ldots \overline{\mathrm{AAC}} . \overline{\mathrm{AACTC}} \cdot \mathrm{TG} \ldots \ldots \mathrm{AT} \ldots \mathrm{A} \ldots \mathrm{T}$ & $\ldots \ldots \ldots$.... & \\
\hline$\ldots \ldots \ldots \ldots \overline{\mathrm{AAC}} \cdot \overline{\mathrm{AACTC}} \cdot \mathrm{TG} \ldots \ldots \mathrm{AT} \ldots \mathrm{A} \ldots \mathrm{T}$ & $\ldots \ldots \ldots$.... & \\
\hline 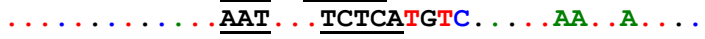 & $\ldots \ldots \ldots$... & \\
\hline$\ldots \ldots \ldots \ldots . \overline{\mathrm{AAT}} \ldots \overline{\text { TCTCA }}$ TGTC . . . AА . А. & $\ldots \ldots \ldots$... & \\
\hline$\ldots \ldots \ldots \ldots . \overline{A A T} \ldots . \overline{T C T C A T G T C} \ldots \ldots$ AA . . . & $\ldots \ldots \ldots \ldots$. & \\
\hline . ТСТСАТGTC..... АА. .А. & $\ldots \ldots \ldots$... & \\
\hline . ТСТСАТGTC. . . . АА. .А. & $\ldots \ldots \ldots$... & \\
\hline . TСTCATGTC...... AA. .A. & $\ldots \ldots \ldots$.... & \\
\hline 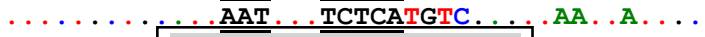 & $\ldots \ldots \ldots$... & \\
\hline . . . . . ATCGTCGCATCTCTTGCTGAG.|GCT.CA. & $\ldots \ldots \ldots .$. & \\
\hline .......... СGTCGCATCTC . TG . . . GCT . CG . T. & $\ldots \ldots \ldots \ldots$. & \\
\hline . . СGTCGCATCTC.TG. . . . . GCT.CG. . T. & ...A. & \\
\hline . CGTCGCATCTC. TG. ..... GCT.CG. . T. & $\ldots \ldots \ldots$ A. & \\
\hline . . . СGTCGCATCTC.TG. . ... GCT.CG. . T. & $\ldots \ldots \ldots \ldots$.... & $\bullet$ \\
\hline$\ldots \ldots \ldots \ldots$. $\ldots$.T. CATCTC.TGT. & $\cdots \cdots \cdots$ & \\
\hline 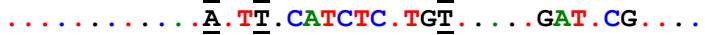 & $\ldots \ldots \ldots$ A. & \\
\hline 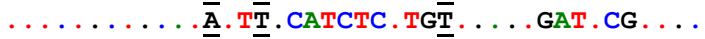 & $\ldots \ldots \ldots$... & - . \\
\hline 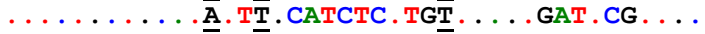 & $\ldots \ldots \ldots$. & \\
\hline$\ldots \ldots \ldots . \overline{\mathrm{A}} \cdot \mathrm{T} \overline{\mathrm{T}} \cdot \mathrm{CATCTC} \cdot \mathrm{TG} \overline{\mathrm{T}}$. & $\ldots \ldots \ldots$. & \\
\hline
\end{tabular}

Type D (pink)

*Type A

Figure 2: Alignment of novel T. buffeli-like (blue) and T. sinensis (brown) 18S rRNA gene sequences with known T. buffeli sequences (black, green and pink), showing V1 variable (A) and V4 hypervariable (B) regions of the gene. RLB probes were designed from the V4 hypervariable region (B). Blocks indicate sequences from which the RLB probes were designed. Nucleotide differences in the probe sequences within the different genotypes are underlined. 
AEGP/69/ITS AEGP/65/ITS AEGP/66/ITS AEGP/73/ITS AEGP/ 74 / ITS

AEGP/69/ITS

AEGP/65/ITS AEGP/66/ITS AEGP/73/ITS AEGP/74/ITS

AEGP/69/ITS AEGP/65/ITS AEGP/66/ITS AEGP/73/ITS AEGP/74/ITS

AEGP/69/ITS AEGP/65/ITS AEGP/66/ITS AEGP/73/ITS AEGP/74/ITS

$\begin{array}{llllllllll}110 & 120 & 130 & 140 & 150 & 160 & 170 & 180 & 190 & 200\end{array}$

$\ldots|\ldots| \ldots|\ldots| \ldots|\ldots| \ldots|\ldots| \ldots|\ldots| \ldots|\ldots| \ldots|\ldots| \ldots|\ldots| \ldots|\ldots| \ldots|\ldots| \ldots|\ldots| \ldots|\ldots|$

$\begin{array}{llllllllll}210 & 220 & 230 & 240 & 250 & 260 & 270 & 280 & 290 & 300\end{array}$ $\ldots|\ldots| \ldots|\ldots| \ldots|\ldots| \ldots|\ldots| \ldots|\ldots| \ldots|\ldots| \ldots|\ldots| \ldots|\ldots| \ldots|\ldots| \ldots|\ldots| \ldots|\ldots| \ldots|\ldots| \ldots \mid$ GAGTGATGACCTCCCAGGGTCATTGTTTCTAGTTAAACTGGTGTCTGTGTGCACGGCCACTTTACGTGGTGTGGAACTTATGATGTAACTTGTTACTCGC

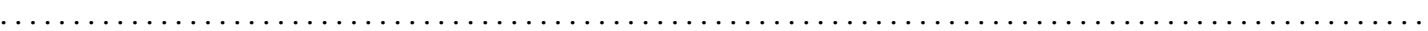

$\ldots|\ldots| \ldots|\ldots| \ldots|\ldots| \ldots|\ldots| \ldots|\ldots| \ldots|\ldots| \ldots|\ldots| \ldots|\ldots| \ldots|\ldots| \ldots|\ldots| \ldots|\ldots| \ldots|\ldots| \ldots \mid \ldots$ GTTCTACTTTGTGCTACACTTTCACATAGCTTAACATCCCTGTCTTTATGACGTGTCACTTCTGCCTGTTTGGCGGTTGTGGATAACGCGGAGGGATTTT

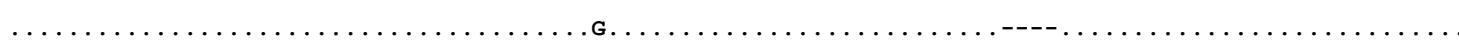$$
\text { .c. }
$$$$
\text { G }
$$

$\begin{array}{llllllll}410 & 420 & 430 & 440 & 450 & 460 & 470 & \mathbf{4 8 0}\end{array}$

$\ldots|\ldots| \ldots|\ldots| \ldots|\ldots| \ldots|\ldots| \ldots|\ldots| \ldots|\ldots| \ldots|\ldots| \ldots|\ldots| \ldots|\ldots| \ldots|\ldots| \ldots \mid \ldots$

AEGP/69/ITS AATTTTAGTTCCTGAGATTGGGTGAGACTATCCACTGAATTTAAACATATAATTAAGTGGAAGAAAAGAAAATAAATATGATTCCCC

AEGP/65/ITS

AEGP/66/ITS

AEGP/73/ITS

AEGP/74/ITS

Figure 3: Sequence alignment of the complete ITS2 region of novel Theileria spp. from the Addo Elephant Game Park.

The dots indicate conserved nucleotides; gaps (-) indicate missing nucleotides and were introduced to maintain homology. 
Amplicons from five out of seven samples from AEGP were successfully sequenced. The sequence alignment of the AEGP ITS sequences (Genbank accession nos. $\underline{\text { JQ0377791, }} \underline{\text { JQ0377792. }}$. $\underline{\mathbf{J Q 0 3 7 7 7 9 3}}, \underline{\mathbf{J Q 0 3 7 7 7 9 4}}, \underline{\mathbf{J Q 0 3 7 7 9 5}}$ ) is indicated in Figure 3. The sequences differed in the lengths of their complete ITS region (952 - $1173 \mathrm{bp})$, as well as in the ITS1 (458 - $642 \mathrm{bp})$ and ITS2 (297 $344 \mathrm{bp}$ ) regions. The $5.8 \mathrm{~S}$ gene was conserved amongst the sequences and shorter (187 bp) than the ITS1 and ITS2 regions. Unlike the novel 18S rRNA gene sequences which were identical, there was polymorphism among the novel ITS sequences, with most of the variation occurring in the ITS1 region. This was mainly due to insertions or deletions of blocks of sequences as seen with the sequences of the ITS2 region (Figure 3). Three sequences (AEPG/65/ITS, AEPG/66/ITS, AEPG/69/ITS) were identical, while sequences AEPG/73/ITS and AEPG/73/ITS differed from each other at 4 positions, and differed from the other samples by $134 \mathrm{bp}$. There was extensive variation between these sequences and the homologous T. buffeli ITS sequences from China (107 - 164 bp), USA and Japan (159 - $200 \mathrm{bp})$, and T. sinensis ITS sequences (144 - $178 \mathrm{bp})$. These differences were in concordance with those obtained from analyses of similar genotypes/species of the $18 \mathrm{~S}$ rRNA gene.

A representative tree generated by Bayesian inference is shown in Figure 4. Three distinct clusters of the T. buffeli sequences were observed in all the trees. Cluster 1 was composed of the T. sergenti and T. buffeli sequences from the USA and Japan (Chitose). These two groups share identical $18 \mathrm{~S}$ rRNA sequences (Figure 2, Chae et al., 1998, 1999a; Aktas et al., 2007) but different ITS (Figure 5; Aktas et al., 2007) and MPSP (Gubbels et al., 2000) sequences.

Cluster 2 was composed of the novel ITS sequences from AEGP and the T. buffeli ITS sequences from China. The latter clade is probably synonymous to that of the unclassified $18 \mathrm{~S}$ rRNA sequences which also grouped together with AEGP 18S rRNA sequences (Figure 2). Cluster 3 was that of the $T$. sinensis ITS sequences (Figure 5). The ITS sequences of T. cervi, $T$. uilenbergi and $T$. luwenshuni always grouped together, while the 18S rRNA gene sequences of these species grouped 


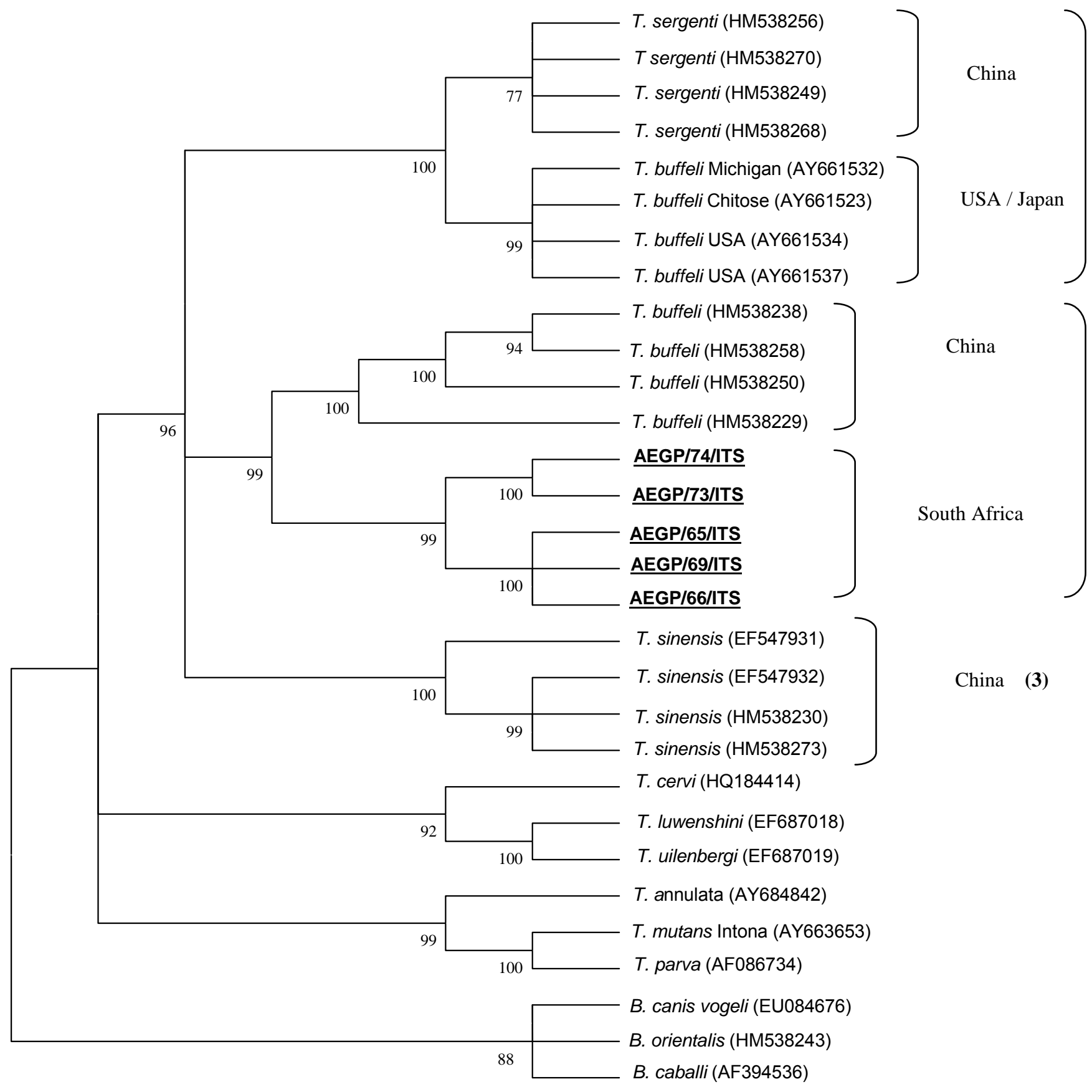

Figure 4: Phylogenetic relationships of novel T. buffeli-like ITS sequences from South Africa (underlined) with known T. buffeli-like ITS sequences from Genbank (accession numbers in parenthesis) as determined by Bayesian inference. The numbers in brackets are designated cluster numbers. Posterior probabilities are indicated at the nodes of the tree. 
separately. Unlike with the 18S rRNA sequences (Figure 2), the T. mutans ITS sequence grouped together with the T. parva and T. annulata sequences (Figure 4; Aktas et al., 2007).

\section{Discussion}

\subsection{Identification of T. buffeli-like and T. sinensis-like 18S rRNA genotypes}

Theileria buffeli was identified as the most commonly occurring species in buffalo in the HIP, mainly co-occurring with other Theileria spp. (Chaisi et al., 2011), and was the only Theileria sp. infecting buffalo in the AEGP. In contrast, we did not identify T. buffeli in buffalo samples from the Kruger National Park (KNP), Greater Limpopo Transfrontier Park (GLTP) and a private game farm bordering the KNP (Chaisi et al., 2011). This species was also identified as the most common Theileria spp. infecting cattle in Tunisia, and was more frequently identified in the sub-humid zone of the country than from the other climatic zones (M'ghirbi et al., 2008).

Our study has revealed that extensive variation exists between the $18 \mathrm{~S}$ rRNA gene sequences of $T$. buffeli of the African buffalo and homologous sequences of T. buffeli from Asian buffalo (Bubalus bubalis) and cattle. In a recent study, Mans et al. (2011) identified T. sinensis-like 18S rRNAgene sequences (which they designated as T. buffeli type-D like) from buffalo samples originating from the Limpopo and KwaZulu-Natal provinces in South Africa, T. buffeli type-C like sequences from buffalo in Mozambique and Limpopo, and T. buffeli type B-like sequences from buffalo in Limpopo and Mozambique.

The distribution of different T. buffeli 18S rRNA genotypes (A, B, C, D, E, H) in buffalo and cattle has previously been reported from other parts of the world by several authors (Chae et al., 1998; 1999a, Chansiri et al., 1999, Gubbels et al., 2000; 2002). The initial classification of these genotypes was based on a $200 \mathrm{bp}$ fragment of the V4 hypervariable region of the 18S rRNA gene (Chae et al., 1998). Type A and D-like organisms have been associated with bovine theileriosis in Missouri (Stockham et al., 2000), Texas (Chae et al., 1999b) and Michigan (Cossio-Bayugar et al., 2002). 
There was extensive variation between $T$. buffeli type D sequences and those of the other $T$. buffeli types. This observation is in agreement with previous studies on the analyses of the 18S rRNA gene and MSPS gene sequences (Chansiri et al., 1999; Gubbels et al., 2000; Yin et al. 2004; Liu et al., 2010b). These authors further indicated that T. buffeli type D organisms may be genetically intermediate between the well-characterized pathogenic Theileria spp. (T. annulata, T. parva, T. lestoquardi, T. uilenbergi, T. luwenshuni) and the benign T. buffeli/T. orientalis spp. Maximum likelihood and parsimony analysis of $18 \mathrm{~S}$ rRNA gene sequences of Theileria spp. by Chanisiri et al. (1999) grouped the T. buffeli type D sequences with the pathogenic Theileria spp., whereas distance methods grouped them with those of the other $T$. buffeli sequences. Additionally, randomly amplified polymorphic DNA (RAPD) profiles generated from Theileria sp. Thung Song (a type D sequence), were different from those of the other benign T. buffeli-like species (Chansiri et al., 1999). For these reasons, these authors indicated that the classification of $T$. buffeli type D species is questionable and should be investigated. Bai et al. (1995) identified a sequence from cattle in China that was similar to the $18 \mathrm{~S}$ rRNA sequences of $T$. buffeli type D and Theileria sp. Thung Song. After studying the morphology, vector and phylogenetic relationship of this novel genotype with other Theileria spp., this genotype was found to be a distinct species and was designated as $T$. sinensis (Bai et al., 2002a, b).

Together with $T$. annulata, $T$. sinensis and $T$. sergenti are the causative agents of bovine theiloriosis in China (Liu et al., 2010b). Morphologically, T. sinensis and T. sergenti are indistinguishable (Yin et al., 2002), but they have different tick vectors as T. sergenti is transmitted by Haemaphysalis longicornis (Liu et al., 2010b). Liu et al. (2010b) developed a PCR assay, based on MPSP gene sequences, for the detection and discrimination of these two species from cattle and yak.

The presence of organisms with $T$. sinensis-like $18 \mathrm{~S}$ rRNA gene sequences in the African buffalo could be of significant importance, particularly to the cattle industry in South Africa as buffalo might act as a source of infection, via infected ticks, to naïve cattle. However, there are currently no reported cases of theileriosis that have been attributed to T. buffeli in South Africa. The vectors of 
both T. buffeli-like and T. sinensis-like genotypes of the South African buffalo are unknown, and should be investigated.

\subsection{Identification of novel 18S rRNA gene sequences by the RLB hybridization assay}

The $T$. buffeli RLB hybridization assay probe that was used in the identification of $T$. buffeli from buffalo (Chaisi et al., 2011) was designed by Gubbels et al. (1999) and it can detect rDNA of all known T. buffeli-like genotypes. Hence, all the samples that we characterized had tested positive for T. buffeli by this assay. Subsequently, Gubbels et al. (2000) designed additional RLB probes for the specific detection of Type D, non-type D, and Type A genotypes, and another probe that detected all the other known T. buffeli 18S rRNA genotypes (Ikeda, B, C, E, H, Warwick).

The novel T. buffeli genotype that we identified from buffalo in South Africa, and the unclassified genotype that was identified from buffalo and cattle in China and India, are all non type-D genotypes but they will not be detected by the non type-D probe due to the nucleotide differences ( 4 $-7 \mathrm{bp}$ ) in the RLB probe sequence. A new non type-D probe can be designed in a different area to include the detection of these novel variants from buffalo in South Africa. Additional probes can also be designed for the specific detection of type SA1 and SA2 genotypes in cattle and buffalo in South Africa.

\subsection{Theileria buffeli-like ITS genotypes}

Theileria parva, T. annulata, T. mutans, T. ovis, T.sergenti and T. buffeli/orientalis have previously been studied at this locus (Collins and Allsopp, 1999; Bendele, 2005; Aktas et al., 2007; Kamau et al., 2011). As observed in our study, the two spacer regions (ITS1 and ITS2) were highly polymorphic in both length and nucleotide composition, and the $5.8 \mathrm{~S}$ region is highly conserved between sequences of related species and is shorter than the spacer regions. Our results also indicate a closer evolutionary relationship between T. mutans and T. parva at this locus as previously indicated by Aktas et al., 2007. Minor polymorphism occurring in a single sequence is possibly due to Taq polymerase error but nucleotide differences occurring in more than one sequence are 
regarded as real (Zahler et al., 1998; Aktas et al., 2007). We therefore regard the variations that we observed as real as the variations were observed in more than one sequence.

Aktas et al. (2007) indicated that there were more variations in the ITS sequences of the pathogenic T. annulata, than in the mildly pathogenic T. mutans and T. sergenti, or benign T. buffeli/orientalis. The genetic variation in pathogenic species may be due to the presence of mixed parasite populations within isolates or to the ingestion of greater numbers of organisms by ticks during the acute phase of the disease, leading to a greater chance of recombination during gametogenesis (Collins and Allsopp, 1999; Aktas et al., 2007). We could not make a comparison of the variation between the different Theileria species as our study was based only on the T. buffeli/orientalis group.

As was the case with the $18 \mathrm{~S}$ gene sequences from HIP clones, we expected the cloned ITS sequences from samples from HIP to group together with the $T$. sinensis ITS sequences from China. However, this was not the case as five of the ITS sequences grouped together with T. parva and T. mutans sequences and two ITS sequences were more similar to the $T$. buffeli-like ITS sequences from AEGP. It is therefore possible that buffalo in HIP harbour both T. buffeli and T. sinensis-like ITS genotypes, however, a lot more sequence data is required to verify this speculation. The occurrence of $T$. parva and/or T. mutans sequences in samples that had tested negative for these species by RLB probably indicated parasitemia that was below the detection limit of the assay. This also confirms the complexity of identification of Theileria spp. in mixed infections in buffalo as we previously reported (Chaisi et al., 2011).

\subsection{Classification of the novel Theileria spp. genotypes}

Based on the phylogenetic positions, nucleotide differences (with known sequences) in the fulllength sequences and the hypervariable (V4) region of the 18S rRNA gene and ITS region, it is possible that the two novel Theileria spp. genotypes from the South African buffalo represent distinct species. However, additional molecular and biological data are required for such 
classification. It is also not clear if the genetic distances within the $18 \mathrm{~S}$ rRNA gene sequences of Types B, C, E, H, Ikeda, Ipoh and Medan represent heterogeneity within the same or different Theileria spp. (Chae et al., 1999a), and there is currently no consensus in the classification of novel genotypes as new species based on the number of nucleotide differences. Our results indicate that the $18 \mathrm{~S}$ rRNA sequences of these genotypes form a monophyletic group that is separated from the other T. buffeli genotypes.

In conclusion, we have established the phylogenetic position of $T$. buffeli-like organisms occurring in buffalo in the AEGP based on 18S rRNA gene and ITS sequence, and that of $T$. sinensis-like organisms of buffalo in the HIP. This study has confirmed that T. buffeli is a highly diverse and cosmopolitan species. The role of buffalo and other wildlife as reservoir hosts of these species should be investigated as buffalo are known to be sources of many infectious diseases of cattle in South Africa (Mashishi, 2002). Future studies should focus on animal transmission studies in order to determine the tick vectors of the $T$. buffeli-like and $T$. sinensis-like genotypes, and on epidemiological studies using new probes that specifically detect and differentiate these novel genotypes in hosts and tick vectors in South Africa. This study therefore provides useful genetic information towards the proper classification of this very complex group.

\section{Acknowledgements}

This work was part of a $\mathrm{PhD}$ project that was funded by the South African National Research Foundation (NRF ICD2006072000009) and UP Research Development Programme. It also falls under the Belgian Directorate General for Development Co-operation Framework agreement ITM/DGCD. We thank Lan He for her assistance in the lab and Milana Troskie for providing the DNA of the AEGP samples. Buffalo blood samples were provided by Drs Roy Bengis, Fred Potgieter and Dave Cooper. 


\section{References}

Aktas, M., Bendele, K.G., Altay, K., Dumanli, N., Tsuji, M., Holman, P.J., 2007. Sequence polymorphism in the ribosomal DNA internal transcribed spacers differs among Theileria species. Vet. Parasitol. 147, 221-230.

Allsopp, M.T., Cavalier-Smith, T., De Waal, D.T., Allsopp, B.A., 1994. Phylogeny and evolution of the piroplasms. Parasitology 108, 147-152.

Altay, K., Aydın, M.F., Dumanlı, N., Aktas, M., 2008.Molecular detection of Theileria and Babesia infections in cattle. Vet. Parasitol. 158, 295-301.

Altschul, S.F., Gish, W., Miller, W., Myers, E.W.,Lipman, D.J., 1990. Basic local alignment search tool. J. Mol. Biol. $215,403-410$

Bai, Q., Liu, G., Hen, G., 1995.An unidentified Theileria species infective for cattle discovered in China. Chin.J. Vet. Sci. $15,15-21$.

Bai, Q., Liu, G.Y., Yin, H., Zhao, Q.Z., Liu, D.K., Ren, J.X., Li, X., 2002a. Theileria sinensis sp. nov.: study on classical taxonomy. Acta Vet. Zootech. Sin.33, 73-77.

Bai, Q., Liu, G.Y., Yin, H., Zhao, Q.Z., Liu, D.K., Ren, J.X., Li, X., 2002b.Theileria sinensis nov: study on molecular taxonomy. Acta Vet. Zootech. Sin. 33, 185-190.

Bendele, K.G., 2005. Molecular characterization of Theileria spp.using ribosomal RNA. MSc. Thesis: Texas A \& M University, USA.

Bonfield, J.K., Smith, K., Staden, R., 1995. A new DNA sequence assembly program. Nucleic Acids Res. 23, 49924999.

Bosman, A.M., Oosthuizen, M.C., Peirce, M.A., Venter, E.H.,Penzhorn, B.L., 2010. Babesialengau sp. nov., a novel Babesia species in cheetah (Acinonyx jubatus, schreber, 1775) populations in south africa. J. Clin. Microbiol. 48, 2703-2708.

Chae, J., Lee, J., Kwon, O., Holman, P.J., Waghela, S.D., Wagner, G.G., 1998.Nucleotide sequence heterogeneity in the small subunit ribosomal RNA gene variable (V4) region among and within geographic isolates of Theileria from cattle, elk and white-tailed deer. Vet. Parasitol. 75, 41-52. 
Chae, J.S., Allsopp, B.A., Waghela, S.D., Park, J.H., Kakuda, T., Sugimoto, C., Allsopp, M.T., Wagner, G.G., Holman, P.J., 1999a. A study of the systematics of Theileria spp. based upon small-subunit ribosomal RNA gene sequences. Parasitol. Res. 85, 877-883.

Chae, J., Levy, M., Hunt, J. Jr., Schlater, J., Snider, G., Waghela, S.D., Holman, P.J., Wagner, G.G., 1999b. Theileria sp. infections associated with bovine fatalities in the United States confirmed by small-subunit rRNA gene analyses of blood and tick samples. J. Clin. Microbiol. 37, 3037-3040.

Chae, J.S., Waghela, S.D., Craig, T.M., Kocan, A.A., Wagner, G.G., Holman, P.J., 1999c. Two Theileriacervi SSU RRNA gene sequence types found in isolates from white-tailed deer and elk in North America. J. Wildl. Dis. 35, 458-465

Chaisi, M.E., Sibeko, K.P., Collins, N.E.,Potgieter, F.T., Oosthuizen, M.C., 2011. Identification of Theileriaparva and Theileria sp. (buffalo) 18S rRNA gene sequence variants in the African buffalo (Syncerus caffer) in southern Africa. Vet. Parasitol. 182, 150-162.

Chansiri, K., Kawazu, S.I., Kamio, T., Terada, Y., Fujisaki, K., Philippe, H., Sarataphan, N., 1999. Molecular phylogenetic studies on Theileria parasites based on small subunit ribosomal RNA gene sequences. Vet. Parasitol. 83, 99-105.

Collins, N.E.,Allsopp, B.A., 1999. Theileria parva ribosomal internal transcribed spacer sequences exhibit extensive polymorphism and mosaic evolution: Application to the characterization of parasites from cattle and buffalo. Parasitology 118, 541-551.

Cossio-Bayugar, R., Pillars, R., Schlater, J., Holman, P.J., 2002.Theileria buffeli infection of a Michigan cow confirmed by small subunit ribosomal RNA gene analysis. Vet. Parasitol. 105, 105-110.Criado, A., Martinez, J., Buling, A., Barba, J.C., Merino, S., Jefferies, R., Irwin, P.J., 2006. New data on epizootiology and genetics of piroplasms based on sequences of small ribosomal subunit and cytochrome b genes. Vet. Parasitol. 142, 238-247.

De Rojas, M., Ubeda, J.M., Cutillas, C., Mora, M.D., Ariza, C., Guevara, D., 2007. Utility of ITS1-5.8S-ITS2 identification and phylogenetic inference within the Rhinonyssus coniventries species complex (Acari: Rhinonyssidae). Parasitol. Res. 100, 1041-1046.

Fujisaki, K., Kawazu, S., Kamio, T., 1994. The taxonomy of the bovine Theileria spp. Parasitology Today 10, 31-33. 
Fuujisaki, K., 1992. 1992. A review of the taxonomy of Theileria sergenti/buffeli/orientalis group parasites in cattle. J. Protozool. Research 2, 87-96.

Gimenez, C., Casado, N., Criado-Fornelio, A., Alvarex de Miguel, F., Dominguez-Penafiel, G., 2009.A molecular survey of Piroplasmida and Hepatozoon isolated from domestic and wild animals in Burgos (northern Spain). Vet.Parasitol. 162, 147-150.

Gubbels, J.M., Vos, A.P., Weide, M., Viseras, J., Schouls, L.M., Vries, E., Jongejan, F., 1999. Simultaneous detection of bovine Theileria and Babesia species by reverse line blot hybridization. J. Clin. Microbiol. 37, 1782-1789.

Gubbels, M.J., Hong, Y., Weide, M., Qi, B., Nijman, I.J., GuangYuan, L., and Jongejan, F., 2000.Molecular characterisation of the Theileria buffeli/orientalis group. Int. J. Parasitol. 30, 943-952.

Gubbels, M.J., Yin, H., Bai, Q., Liu, G., Nijman, I.J., and Jongejan, F., 2002.The phylogenetic position of the Theileria buffeli group in relation to other Theileria species. Parasitol. Res. 88, S28-32.

Hall, T.A., 1999.BioEdit: a user-friendly biological sequence alignment editor and analysis program for Windows 95/98/NT. Nucl. Acid S. 41, 95-98.

He, L., Feng, H.H., Zhang, W.J., Zhang, Q.L., Fang, R., Wang, L.X., Tu, P., Zhou, Y.Q., Zhao, J.L., Oosthuizen, M.C., 2012. Occurrence of Theileria and Babesia species in water buffalo (Bubalus babalis, linnaeus, 1758) in the Hubei province, South China. Vet. Parasitol. 186, 490-496.

Hills, D.M., Dixon, M.T., 1991. Ribosomal DNA: molecular evolution and phylogenetic inference. Q. Rev. Biol. 66, 411-453.

Hilpertshauser, H., Deplazes, P., Meli, M.L., Hofmann-Lehmann, R., Lutz, H., and Mathis, A., 2007. Genotyping of Babesia bigemina from cattle from a non-endemic area (Switzerland). Vet. Parasitol. 145, 59-64.

Holman, P.J., Bendele, K.G., Schoelkopf, L., Jones-Witthuhn J., Jones, S.O., 2003. Ribosomal RNA analysis of Babesia odocoilei isolates from farmed reindeer (Rangifer tarandus

tarandus) and elk (Cervus elaphus canadensis) in Winsconsin. Parasitol.Res. 91, 378-383.

Kamau, J., Salim, B., Yokoyama, N., Kinyanjui, P., Sugimoto, C., 2011. Rapid discrimination and quantification of Theileria orientalis types using ribosomal DNA internal transcribed spacers. Infect. Genet. Evol. 11, 407-414. 
Katoh, K., Kuma, K., Toh, H., Miyata, T., 2005. MAFFT version 5: Improvement in accuracy of multiple sequence alignment. Nucl. Acids Res. 33, 511-518.

Kawazu, S., Kamio, T., Kakuda, T., Terada, Y., Sugimoto, C., and Fujisaki, K., 1999. Phylogenetic relationships of the benign Theileria species in cattle and asian buffalo based on the major piroplasm surface protein (p33/34) gene sequences. Int. J. Parasitol. 29, 613-618.

Lew, A.E., Anderson, G.R., Minchin, C.M., Jeston, P.J., Jorgensen, W.K., 2003. Inter- and intra-strain variation and PCR detection of the internal transcribed spacer 1 (ITS-1) sequences of Australian isolates of Eimeria species from chickens. Vet.Parasitol. 112, 33-50.

Liu, Q., Zhou, Y.Q., He, G.S., Oosthuizen, M.C., Zhou, D.N., Zhao, J.L., 2010a. Molecular phylogenetic studies on Theileria spp. isolates (China) based on small subunit ribosomal RNA gene sequences. Trop. Anim. Health Pro. 42, 109-114.

Liu, A., Guan, G., Liu, Z., Liu, J., Leblanc, N., Li, Y., Gao, J., Ma, M., Niu, Q., Ren, Q., Bai, Q., Yin, H., Luo, J., 2010b. Detecting and differentiating Theileria sergenti and Theileria sinensis in cattle and yaks by PCR based on major piroplasm surface protein (MPSP). Exp. Parasitol. 126, 476-481.

Mans, B.J., Pienaar, R., Latif, A.A., Potgieter, F.T., 2011.Diversity in the 18S SSU rRNA V4 hyper-variable region of Theileria spp. in Cape buffalo (Synceruscaffer) and cattle from Southern Africa. Parasitology 1-14.

Mashishi, M.S.K., 2002.Diseases shared by wildlife and livestock. Department of Agriculture, South Africa: http://www.nda.agric.za/docs/Infopaks/wildlife.htm

M'ghirbi, Y., Hurtado, A., Barandika, J.F., Khlif, K., Ketata, Z., Bouattour, A., 2008. A molecular survey of Theileria and Babesia parasites in cattle, with a note on the distribution of ticks in Tunisia. Parasitol. Res. 103, 435-442.

Morzaria, S.P., Young, A.S., Kimber, C.D., 1977. The serological relationship of a British Theileria with other Theileria species using the indirect fluorescent antibody test. Res. Vet. Sci. 22, 330-333.

Nijhof, A.M., Penzhorn, B.L., Lynen, G., Mollel, J.O., Morkel, P., Bekker, C.P.J., Jongejan, F., 2003.Babesiabicornis sp. nov.andTheileriabicornis sp. nov.: tick-borne parasites associated with mortality in the black rhinoceros (Dicerosbicornis). J. Clin. Microbiol. 41, 2249-2254. 
Niu, Q., Luo, J., Guan, G., Liu, Z., Ma, M., Liu, A., Gao, J., Ren, Q., Li, Y., Qiu, J., Yin, H., 2009. Differentiation of two ovine Babesiabased on the ribosomal DNA internal transcribed spacer (ITS) sequences. Exp.Parasitol. 121, 64-68.

Oosthuizen, M.C., Zweygarth, E., Collins, N.E., Troskie, M., Penzhorn, B.L., 2008.Identification of a novel Babesia sp. from a sable antelope (Hippotragusniger Harris, 1838). J. Clin. Microbiol. 46, 2247-2251.

Ronquist, F., Huelsenbeck, J.P., 2003.MrBayes 3: Bayesian phylogenetic inference under mixed models. Bioinformatics $19,1572-1574$.

Saito-Ito, A., Takada, N., Ishiguro, F., Fujita, H., Yano, Y., Ma, X., Chen, E., 2008. Detection of Kobe-type Babesia microti associated with Japanese human babesiosis in field rodents in central Taiwan and southeastern mainland China. Parasitology 135, 691.

Saitou, N., Nei, M., 1987. The neighbor-joining method: A new method for reconstructing phylogenetic trees. Mol. Biol. Evol. 4, 406-425.

Sarataphan, N., Kakuda, T., Chansiri, K., Onuma, M., 2003.Survey of benign Theileria parasites of cattle and buffaloes in Thailand using allele-specific polymerase chain reaction of Major Piroplasm Surface Protein Gene. J. Vet. Med. Sci. 65, 133-135.

Staden, R., Beal, K.F., Bonfield, J.K., 2000.The staden package, 1998. Methods Mol. Biol. 132, 115-130.

Steward, N.P., Uilenberg, G., De Vos, A.J., 1996.Review of Australian species of Theileria, with special reference to Theileriabuffeli of cattle. Trop. Anim. Health Pro. 28, 81-90.

Stockham, S.L., Kjemtrup, A.M., Conrad, P.A., Schmidt, D.A., Scott, M.A., Robinson, T.W., Tyler, J.W., Johnson, G.C., Carson, C.A., Cuddihee, P., 2000. Theileriosis in a Missouri beef herd caused by Theileriabuffeli: case report, herd investigation, ultrastructure, phylogenetic analysis, and experimental transmission. Vet.Pathol. 37, $11-21$.

Sun, C., Liu, Z., Gao, J., Guan, G., Ma, M., Luo, J., Yin, H., 2008. Investigations into the natural infection rate of Haemaphysalis qunghaiensis with Piroplasma using nested PCR. Exp. Appl. Acarol. 4, 107-114. 
Swofford, D.L., 2003.PAUP*.Phylogenetic Analysis Using Parsimony (*and other methods).Version 4b10.Sinauer Associates, Sunderland, Massachusetts.

Tamura, K., Dudley, J., Nei, M., Kumar, S., 2007. MEGA4: Molecular evolutionary genetics analysis (MEGA) software version 4.0. Mol. Biol. Evol. 24, 1596-1599.

Thompson, J.R., Marcelino, L.A., Polz, M.F., 2002. Heteroduplexes in mixed-template amplifications: Formation, consequence and elimination by 'reconditioning PCR'. Nucleic Acids Res. 30, 2083-2088.

Uilenberg, G., Perie, N.M., Spanjer, A.A., Franssen, F.F., 1985.Theileria orientalis, a cosmopolitan blood parasite of cattle: Demonstration of the schizont stage. Res. Vet. Sci. 38, 352-360.

Uilenberg, G., 2011. Theileria sergenti. Vet. Parasitol. 175, 386.

Yin, H., Guan, G., Ma, M., Luo, J., Lu, B., Yuan, G., Bai, Q., Lu, C., Yuan, Z., Preston, P., 2002.Haemaphysalis qinghaiensis ticks transmit at least two different Theileria species: one is infective to yaks, one is infective to sheep. Veterinary Parasitology 107, 29-35.

Yin, H., Luo, J., Schnittger, L., Lu, B., Beyer, D., Ma, M., Guan, G., Bai, Q., Lu, C., Ahmed, J., 2004. Phylogenetic analysis of Theileria species transmitted by Haemaphysalis qinghaiensis. Parasitol. Res. 92, 36-42.

Wang, L.X., He, L., Fang, R., Song, Q.Q., Tu, P., Jenkins, A., Zhou, Y.Q., Zhao, J.L., 2010.Loop-mediated isothermal amplification (LAMP) assay for detection of Theileria sergenti infection targeting the p33 gene. Veterinary Parasitology 171, 159-162.

Zahler, M., Schein, E., Rinder, H., and Gothe, R., 1998. Characteristic genotypes discriminate between Babesia canis isolates of differing vector specificity and pathogenicity to dogs. Parasitol. Res. 84, 544-548. 